\title{
BMJ Open Impact of virtual care on health-related quality of life in children with diabetes mellitus: a systematic review protocol
}

\author{
Raeesha Rajan (D , , ${ }^{1,2}$ Maya Kshatriya, ${ }^{1,3}$ Laura Banfield, ${ }^{4}$ Uma Athale, ${ }^{1,5}$ \\ Lehana Thabane (D) , ${ }^{2}$ M Constantine Samaan (i) ${ }^{1,3,6,7}$
}

To cite: Rajan R, Kshatriya M, Banfield L, et al. Impact of virtual care on health-related quality of life in children with diabetes mellitus: a systematic review protocol. BMJ Open 2022;12:e053642. doi:10.1136/ bmjopen-2021-053642

- Prepublication history and additional supplemental material for this paper are available online. To view these files, please visit the journal online (http://dx.doi.org/10.1136/ bmjopen-2021-053642).

Received 22 May 2021 Accepted 24 January 2022

Check for updates

(c) Author(s) (or their employer(s)) 2022. Re-use permitted under CC BY-NC. No commercial re-use. See rights and permissions. Published by BMJ.

For numbered affiliations see end of article.

Correspondence to Dr M Constantine Samaan; samaanc@mcmaster.ca

\section{ABSTRACT}

Introduction Diabetes mellitus is the most common endocrine disorder in children, and the prevalence of paediatric type 1 and type 2 diabetes continue to rise globally. Diabetes clinical care programs pivoted to virtual care with the COVID-19 pandemic-driven social distancing measures. Yet, the impact of virtual care on health-related quality of life in children living with diabetes remains unclear. This protocol reports on the methods that will be implemented to conduct a systematic review to assess the health-related quality of life and metabolic health impacts of virtual diabetes care.

Methods and analysis We will search MEDLINE, Embase, EMCare, PsycInfo, Web of Science, and the grey literature for eligible studies. We will screen title, abstract, and fulltext papers for potential inclusion and assess the risk of bias and the overall confidence in the evidence using the Grading of Recommendations, Assessment, Development, and Evaluation (GRADE) approach. A meta-analysis will be conducted if two studies report similar populations, study designs, methods, and outcomes.

This systematic review will summarise the health-related quality of life outcomes for virtual diabetes care delivery models.

Ethics and dissemination No ethics approval is required for this systematic review protocol as it does not include patient data. The systematic review will be published in a peer-reviewed journal and presented at international conferences.

PROSPERO registration number CRD42021235646.

\section{INTRODUCTION}

Diabetes mellitus is the most common endocrinopathy in children. ${ }^{1}$ Over 1.1 million children and youth are living with type 1 diabetes mellitus (T1DM) globally. ${ }^{23}$ While the global prevalence of type 2 diabetes mellitus (T2DM) has not been fully measured, the prevalence rates are estimated to be up to 5330/100 000 children, ${ }^{4}$ and paediatric T2DM rates are steadily rising in parallel with its main driver, obesity. $^{25}$

Diabetes care and outcomes have been transformed over the past few decades with the development of new insulins, insulin delivery systems, and glucose monitoring

\section{Strengths and limitations of this study}

This systematic review protocol includes a detailed reporting of the methods to assess health-related quality of life outcomes with virtual diabetes care in paediatric patients, and guides future policy directions in implementing virtual care in this population.

- The use of virtual care models and their impact on diabetes outcomes is a relatively novel area of care, and having data on their impact is critical to justify scalability.

If there is high heterogeneity, it will not be feasible to conduct a meta-analysis to estimate effect size.

devices. $^{6-12}$ In addition, outpatient-based multidisciplinary care have become the norm, and several guidelines recommend quarterly clinic visits .6 710111314 This care approach, while beneficial, is labor-intensive, costly, and can be disruptive to caregivers' work schedules and school time for children, with a negative impact on health-related quality of life (HRQOL). ${ }^{131516}$

Since the start of the COVID-19 pandemic, a seismic shift in diabetes care took place; multidisciplinary care needed to shift swiftly to virtual platforms due to social distancing measures to protect patients and manage finite healthcare resources. ${ }^{17} 18$ While it is unclear whether virtual care has had a negative impact on outcomes, the full implications of this shift in care delivery are starting to emerge. $^{18}$

Virtual care for diabetes during the COVID-19 pandemic is building on existing trends of increased access to remote care using web-based (eHealth) and mobilebased (mHealth) platforms. These platforms included telephone, Skype for Business, and Zoom that allow audio and video communications between patients, families, and healthcare providers. ${ }^{17} 19$ The online platforms of continuous glucose monitoring devices and insulin pumps were leveraged 
for sharing accurate glycaemic control and management data with healthcare providers. ${ }^{17182021}$ eHealth refers to internet, virtual reality, or digital gaming applications that allow users to monitor, manage, or learn more about their health through video, text, or interactive learning media. ${ }^{22-24}$ mHealth refers to portable or wireless applications such as text messaging, mobile-compatible applications (apps), wearable devices, or the use of social media. ${ }^{22} 2325$ The integration of virtual care modalities into healthcare is quite promising due to their relatively low cost, scalability, and association with successful patient engagement, satisfaction with communication with healthcare teams, and the facilitation of access to specialised care and diabetes monitoring. ${ }^{2627}$

While focus on virtual interventions to improve glycaemic control is important, this improvement may impact HRQOL as the responsibilities associated with this care approach may be burdensome for patients and their families. ${ }^{8}$ The eHealth and mHealth approaches increase patient involvement in their care but there is also a need for familiarity with technical aspects of these care modalities. ${ }^{27} 28$ There is currently no existing evidence synthesis of the impact of virtual interventions on HRQOL in children living with diabetes.

The objectives of this systematic review protocol are to report on the methods of a systematic review to determine the effectiveness of eHealth and mHealth interventions in maintaining or improving HRQOL, glycaemic control, overweight and obesity, and assess their impact on adverse events in children living with diabetes. Also, we aim to determine patient and caregiver satisfaction, and the acceptability and feasibility of virtual care modalities.

\section{Research questions}

Primary

In children with diabetes, does the use of eHealth and mHealth interventions, when compared with in-person care, maintain or improve HRQOL?

\section{Secondary}

In children with diabetes, do virtual interventions maintain or improve glycaemic control, body mass and reduce adverse diabetes-related outcomes when compared to in-person care? Is virtual care usable and acceptable for this population?

\section{METHODS}

The methodology for this protocol has been reported based on the Preferred Reporting Items for Systematic Review and Meta-Analysis Protocols (PRISMA-P) guideline ${ }^{29}{ }^{30}$ (online supplemental file 1). This protocol has been registered with PROSPERO. We aim to complete this systematic review between October 2021 and March 2022.

\section{Patient and public involvement}

Patients and/or the public were not involved in the design, conduct, reporting or dissemination plans of this research.

\section{Search strategy}

This review's search strategies will be developed by a Senior Health Sciences Librarian (LB), who has expertise in designing systematic review search strategies. A sample Ovid MEDLINE search strategy is reported in table 1. Our searches will run on the following platforms: Ovid MEDLINE, Ovid Embase (Elsevier), Ovid EMCare (Elsevier), PsycInfo, and Web of Science (Clarivate). The grey literature searches will be done using ProQuest Dissertations and Theses A\&I and ClinicalTrials.gov.

\section{Inclusion criteria}

We will include studies that recruited boys and girls ages 2-18 years, from all ethnicities and diagnosed with T1DM or T2DM. The diagnosis of diabetes is stablished using standardised criteria, with a random or a 2-hour post oral glucose tolerance test $\geq 11.1 \mathrm{mmol} / \mathrm{L}(200 \mathrm{mg} / \mathrm{dL})$, or a fasting glucose level of $\geq 7.0 \mathrm{mmol} / \mathrm{L}(126 \mathrm{mg} / \mathrm{dL}) .{ }^{6}$

Children at all pubertal stages based on Tanner staging, ${ }^{31}{ }^{32}$ glycaemic control levels (using HbAlc) and body mass index (BMI) z-scores will be included. Children treated with insulin injections of all insulin types and regimens (multiple daily injection regimens, two times a day regimens, insulin mixes) will be included. We will also have children on insulin pump therapy regardless of pump type and those using glucometers or continuous glucose sensors as applicable.

We will include studies from all geographic locations, languages, and publication timing.

The eligible study designs to be included in this systematic review are randomised and non-randomised comparative studies. We will exclude protocol papers, case reports, and commentaries. Eligible studies which are not published in English will be translated using google translate.

Children with comorbid autoimmune conditions (e.g. Hashimoto's thyroiditis, Graves' disease, coeliac disease), as well as children with diabetes-related complications including neuropathy, nephropathy, and retinopathy will be included.

This review will include interventions that deliver diabetes care via eHealth and mHealth approaches. Diabetes education and care refers to personalised patient and caregiver engagement to monitor and promote glycaemic control and limit comorbidities. ${ }^{70}$ eHealth $^{10}$ refers to the Internet, virtual reality, or digital gaming applications that allow users to monitor, manage, or learn more about their health through video, text, or interactive learning media. ${ }^{22-24}$ mHealth refers to portable or wireless applications such as text messaging, mobilecompatible applications (apps), wearable devices, or the use of social media. ${ }^{22} 23$ These applications also allow for user engagement with health monitoring, management, and learning. ${ }^{24} 2533$

Comparator groups will be reported if available. These groups will include patients receiving usual care ; this approach includes diabetes education and care delivery via in-person clinic visits with diabetes healthcare teams. 


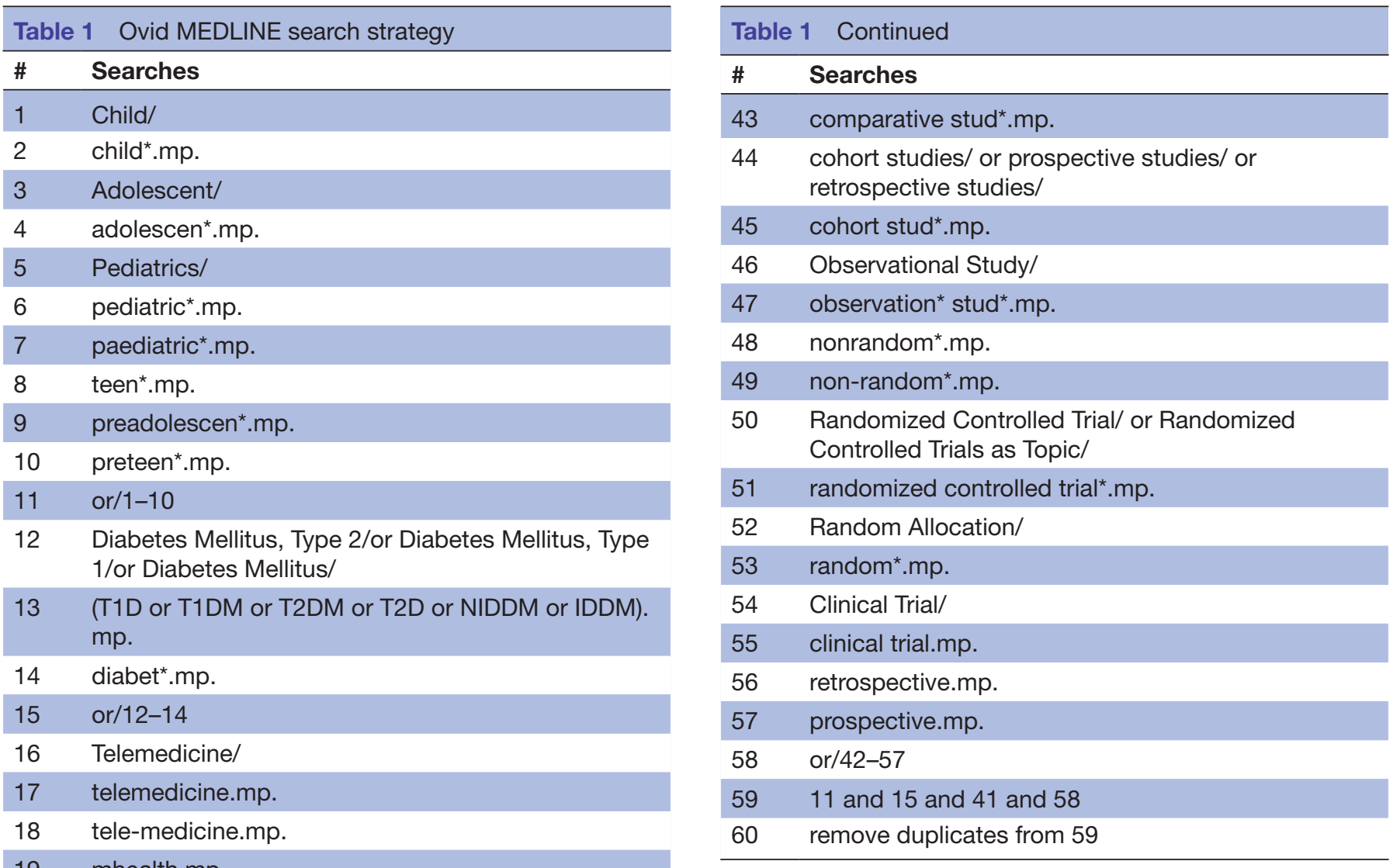

These visits include a review of glucose trends in logbooks or sensor downloads, a review of insulin regimens for injectors and pump settings for children on insulin pumps, dietary guidance, physical activity recommendations, and emotional and mental health screening and supports. ${ }^{15} 19$ Also, information and education about lifestyle factors are included in these visits. ${ }^{15} 19$

We will include studies that report on the HRQOL, measured by diabetes HRQOL questionnaires. These include the Pediatric Quality of Life Inventory ${ }^{34}$ or the Diabetes Quality of Life for Youth Questionnaire. ${ }^{36}$ Other questionnaires, such as the proxy formats of general HRQOL questionnaires for parents and families and others, will also be included. Studies that have failed to report one or more measured outcomes will not be excluded.

\section{Exclusion criteria}

We will exclude studies on boys and girls with other types of diabetes including Maturity Onset Diabetes of the Young, gestational diabetes mellitus, and cystic fibrosisrelated diabetes, among others. Children receiving medications that can drive hyperglycaemia, such as steroids and immunosuppressants, will also be excluded.

\section{OUTCOMES MEASURES}

Critical outcome

This systematic review's critical outcome includes the maintained or improved HRQOL, as measured by 
diabetes HRQOL questionnaires, or proxy formats of the questionnaires used to measure HRQOL.

\section{Important outcomes}

Important outcomes of this study include:

1. Maintained or improved glycaemic control, as measured by HbA1c, logbook glucose readings or glucose sensor-based data, for example, DexCom G6, ${ }^{37}$ FreeStyle Libre II sensors ${ }^{38}$ or others. While the target HbAlc level for people with diabetes is usually $<7.5 \%$ for $\mathrm{T} 1 \mathrm{DM}$ and $\leq 6.5 \%-7 \%$ for T2DM, goals that are adjusted to participants' needs will be accepted. ${ }^{6} 73940$

2. Changes in body mass as measured by assessing BMI z-scores or BMI percentiles pre-intervention and postintervention changes.

3. The number and severity of morbidities. These morbidities include hypoglycaemia (glucose levels $\leq 3.9 \mathrm{mmol} / \mathrm{L}(70 \mathrm{mg} / \mathrm{dL})$ ) and hyperglycaemia (glucose levels $\geq 13.3 \mathrm{mmol} / \mathrm{L}(240 \mathrm{mg} / \mathrm{dL}))$. Also, the occurrence of ketosis, assessed via measurement of blood beta-hydroxybutyrate or urine acetoacetate and using manufacturer-specific reporting measures including quantitative or semiquantitative testing as applicable will be reported. We will also assess rates of diabetic ketoacidosis (defined as hyperglycaemia with blood glucose $>11 \mathrm{mmol} / \mathrm{L}(200 \mathrm{mg} / \mathrm{dL})$, venous blood gasbased $\mathrm{pH}<7.3$, serum bicarbonate $<15 \mathrm{mmol} / \mathrm{L}$ and the presence of ketones ( $\beta$-hydroxybutyrate $\geq 3 \mathrm{mmol} / \mathrm{L}$ in blood, moderate-large ketonuria). ${ }^{61}$ If no specific values were reported to establish the diagnosis of

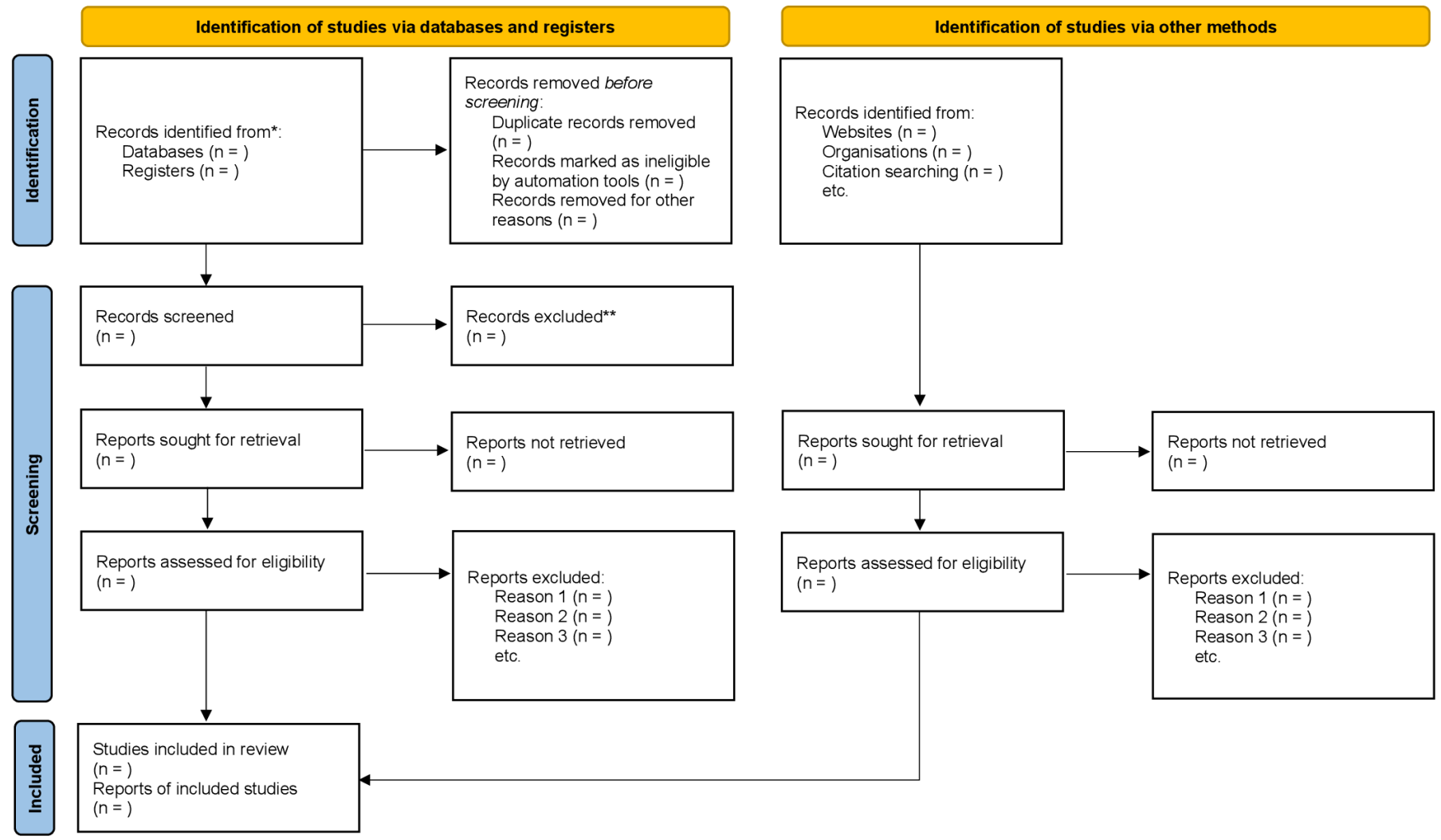

Figure 1 Flow diagram of article screening process.

hypoglycaemia, hyperglycaemia, ketosis, and diabetic ketoacidosis, the above events would be included as a dichotomous outcome.

4. Patient and family satisfaction, and their perceptions of the usability and acceptability of the virtual intervention from relevant questionnaires, which include measures to assess frequency and ease of use and desire for continued application after the study has ended.

\section{Data management}

We will export search results onto Covidence, ${ }^{42}$ and remove duplicates and complete data screening. Data will be abstracted into a Microsoft Excel sheet. We will develop and pilot test the data abstraction form to ensure its validity.

\section{Study selection}

Two reviewers will independently assess the eligibility of titles, abstracts and full-text articles identified in the database searches for potential eligibility and inclusion in this review. Disagreements at each stage will be resolved through discussion among the reviewers. A third reviewer will resolve persistent disagreements. The 2020 PRISMA flow diagram reported in figure 1 will be used to track the process and will be included in the final paper. ${ }^{43} 44$

\section{Data abstraction}

Data from included full-text articles will be abstracted independently by the two reviewers into the standardised data abstraction form. The reviewers will resolve any conflicts 
through discussion, and if necessary, a third reviewer will resolve any persisting discrepancies. We will abstract data including first author's name, country, year, number of participants, age, sex, diabetes type, diabetes duration, comorbidities, insulin type and mode of delivery, and modality of glucose monitoring. Data on hyperglycaemia, hypoglycaemia, ketosis, and diabetic ketoacidosis will also be abstracted. We will abstract relevant data on the interventions including type, duration, details of delivery, HRQOL questionnaires used, HRQOL scores, glycaemic control measures, change in glycaemic control, change in BMI z-score or BMI percentile, adverse events, data on user satisfaction and the usability and acceptability of the interventions. Based on the data available, we will determine whether studies can be pooled for meta-analyses.

We will e-mail the Principal Investigators cited in the publications to obtain any missing data.

\section{Risk of bias assessment}

Two reviewers will independently perform the risk of bias assessments of all randomised controlled trials using the Cochrane RoB 2.0 tool. ${ }^{45}$ Comparative, non-randomised studies will be assessed using the ROBINS-I tool. ${ }^{46}$ Any conflicts will be discussed, and a third reviewer will resolve any remaining disagreements.

\section{GRADE assessment}

To assess the overall confidence in the quality of evidence, we will use the Grading of Recommendations, Assessment, Development, and Evaluation (GRADE) approach. ${ }^{47}$ Quality will be determined by study design, risk of bias, inconsistency, indirectness, imprecision, and publication bias. ${ }^{47}$ The quality of the cumulative evidence and magnitude of the effect will be used to determine the overall strength of the meta-analysis findings if it is feasible to do so.

\section{Statistical analysis}

We will include a summary table of all eligible studies in the final review. A meta-analysis will be conducted if two or more studies of similar study design, methods, populations, interventions, and outcomes are identified. We will analyze the data of randomised trials separately from those of non-randomised comparative studies. We will report dichotomous outcomes as risk ratios or ORs and continuous variables as mean differences or standardised mean differences, with a $95 \%$ CI. A detailed qualitative synthesis of the findings will be provided in a narrative table if a meta-analysis is not possible.

If a meta-analysis is possible, we will perform a randomeffects model which accounts for within-study and between-study variability. We will use the Review Manager (Revman V5.4.1) software to generate forest plots to represent the data graphically if appropriate. ${ }^{48}$

Heterogeneity will be assessed using the $\chi^{2}$ test $p$ value with a cut-off value of 0.1 and the $\mathrm{I}^{2}$ statistic with a cut-off value of $>75 \%$ defined as considerable heterogeneity. ${ }^{49} \mathrm{~A}$ sensitivity analysis will be performed by removing studies with a high risk of bias to assess the impact on the metaanalysis results. ${ }^{50}$

If appropriate, we will conduct subgroup analyses based on the type of diabetes mellitus, as participants with T1DM and T2DM have fundamentally different management goals. We will also perform subgroup analyses based on the age and sex of participants and the mode of insulin administration (injections vs pumps).

If 10 or more studies are included, we will generate a funnel plot and use Egger's test to assess small study effect. Alternatively, we will report publication bias by considering the number of relevant conference abstracts that did not have published articles.

\section{DISCUSSION}

While the number of children and youth living with diabetes is rising globally, the outcomes of these children have improved significantly over the past few decades, thanks to advances in care models and research. ${ }^{17-12}$ However, the burden of care on patients and families is high due to the complex nature of the disease and its treatment, and burnout is a challenge that patients and families struggle with. ${ }^{13} 1516$ With the COVID-19 pandemic, new virtual models of care had to be adopted within a very short period of time to deliver care to these children and youth. ${ }^{17} 18$ While the use of virtual care has grown steadily over the past few years, its impact on HRQOL with diabetes is less well understood compared with their impact on glycaemic control. This systematic review will focus on understanding the impact of virtual diabetes care on HRQOL. As these care approaches now take an unprecedented priority in view of the pandemic, with healthcare systems and policy makers trying and balance safety with the need for delivering quality care worldwide, the data on HRQOL are crucial to help shape the future of virtual diabetes care.

As remote diabetes care and education have become viable alternatives to in-person care in the COVID-19 era, this systematic review will help develop an understanding of its effects on HRQOL outcomes in children living with diabetes, and will guide future directives on implementing virtual diabetes care.

\section{ETHICS AND DISSEMINATION}

Ethics approval is not required as we will not recruit nor include any individual patient data in this systematic review. There are no other ethical and safety considerations. The results of our systematic review will be published in a peer-reviewed journal and presented at international conferences.

\section{Author affiliations}

${ }^{1}$ Department of Pediatrics, McMaster University, Hamilton, Ontario, Canada ${ }^{2}$ Department of Health Research Methods, Evidence, and Impact, McMaster University, Hamilton, Ontario, Canada

${ }^{3}$ Global Health Program, McMaster University, Hamilton, Ontario, Canada ${ }^{4}$ Health Sciences Library, McMaster University, Hamilton, Ontario, Canada 
${ }^{5}$ Division of Hematology Oncology, McMaster Children's Hospital, Hamilton, Ontario, Canada

${ }^{6}$ Division of Pediatric Endocrinology, McMaster Children's Hospital, Hamilton, Ontario, Canada

${ }^{7}$ Michael G. DeGroote School of Medicine, McMaster University, Hamilton, Ontario, Canada

Contributors MCS is the guarantor. RR, MK, LB, UA, LT and MCS were responsible for developing the research question, study conception and design. MCS, LB, RR, MK, UA and LT developed the search strategy and eligibility criteria. RR and MCS were responsible for drafting the manuscript. All authors reviewed, edited and approved the final version of the manuscript.

Funding The authors have not declared a specific grant for this research from any funding agency in the public, commercial or not-for-profit sectors.

Competing interests None declared.

Patient consent for publication Not applicable.

Ethics approval This study does not involve human participants.

Provenance and peer review Not commissioned; internally peer reviewed.

Supplemental material This content has been supplied by the author(s). It has not been vetted by BMJ Publishing Group Limited (BMJ) and may not have been peer-reviewed. Any opinions or recommendations discussed are solely those of the author(s) and are not endorsed by BMJ. BMJ disclaims all liability and responsibility arising from any reliance placed on the content. Where the content includes any translated material, BMJ does not warrant the accuracy and reliability of the translations (including but not limited to local regulations, clinical guidelines, terminology, drug names and drug dosages), and is not responsible for any error and/or omissions arising from translation and adaptation or otherwise.

Open access This is an open access article distributed in accordance with the Creative Commons Attribution Non Commercial (CC BY-NC 4.0) license, which permits others to distribute, remix, adapt, build upon this work non-commercially, and license their derivative works on different terms, provided the original work is properly cited, appropriate credit is given, any changes made indicated, and the use is non-commercial. See: http://creativecommons.org/licenses/by-nc/4.0/.

\section{ORCID iDs}

Raeesha Rajan http://orcid.org/0000-0002-8028-9662

Lehana Thabane http://orcid.org/0000-0003-0355-9734

M Constantine Samaan http://orcid.org/0000-0002-6403-4715

\section{REFERENCES}

1 Green A, Hede SM, Patterson CC, et al. Type 1 diabetes in 2017: global estimates of incident and prevalent cases in children and adults. Diabetologia 2021;64:2741-50.

2 Patterson CC, Karuranga S, Salpea P, et al. Worldwide estimates of incidence, prevalence and mortality of type 1 diabetes in children and adolescents: results from the International diabetes Federation diabetes atlas, 9th edition. Diabetes Res Clin Pract 2019;157:107842.

3 Williams R, Karuranga S, Malanda B, et al. Global and regional estimates and projections of diabetes-related health expenditure: results from the International diabetes Federation diabetes atlas, 9th edition. Diabetes Res Clin Pract 2020;162:108072.

4 Fazeli Farsani S, van der Aa MP, van der Vorst MMJ, et al. Global trends in the incidence and prevalence of type 2 diabetes in children and adolescents: a systematic review and evaluation of methodological approaches. Diabetologia 2013;56:1471-88.

5 World Health Organization. Global report on diabetes, 2020. Available: whoint/news-room/fact-sheets/detail/diabetes

6 American Diabetes Association. 2. Classification and Diagnosis of Diabetes: Standards of Medical Care in Diabetes-2020. Diabetes Care 2020;43:S14-31.

7 Diabetes Canada Clinical Practice Guidelines Expert Committee. Diabetes Canada 2018 clinical practice guidelines for the prevention and management of diabetes in Canada. Can J Diabetes 2018;42:S1-325.

8 Rosner B, Roman-Urrestarazu A. Health-Related quality of life in paediatric patients with type 1 diabetes mellitus using insulin infusion systems. A systematic review and meta-analysis. PLoS One 2019;14:e0217655.

9 Mueller-Godeffroy E, Vonthein R, Ludwig-Seibold C, et al. Psychosocial benefits of insulin pump therapy in children with diabetes type 1 and their families: the pumpkin multicenter randomized controlled trial. Pediatr Diabetes 2018;19:1471-80.

10 American Diabetes Association. 13. Children and Adolescents: Standards of Medical Care in Diabetes-2020. Diabetes Care 2020;43:S163-82.

11 Diabetes Control and Complications Trial Research Group, Nathan DM, Genuth S, et al. The effect of intensive treatment of diabetes on the development and progression of long-term complications in insulin-dependent diabetes mellitus. N Engl J Med 1993;329:977-86.

12 Abraham MB, de Bock M, Smith GJ, et al. Effect of a hybrid closedloop system on glycemic and psychosocial outcomes in children and adolescents with type 1 diabetes: a randomized clinical trial. JAMA Pediatr 2021;175:1227.

13 Gandrud L, Altan A, Buzinec P, et al. Intensive remote monitoring versus conventional care in type 1 diabetes: a randomized controlled trial. Pediatr Diabetes 2018;19:1086-93.

14 Hattersley AT, Greeley SAW, Polak M, et al. ISPAD clinical practice consensus guidelines 2018: the diagnosis and management of monogenic diabetes in children and adolescents. Pediatr Diabetes 2018;19 Suppl 27:47-63.

15 Powers MA, Bardsley J, Cypress M. Diabetes self-management education and support in type 2 diabetes: a joint position statement of the American diabetes association, the American association of diabetes educators, and the Academy of nutrition and dietetics. The Diabetes Educator 2017:43:40-53.

16 Katz ML, Laffel LM, Perrin JM, et al. Impact of type 1 diabetes mellitus on the family is reduced with the medical home, care coordination, and family-centered care. J Pediatr 2012;160:861-7.

17 Fung A, Irvine M, Ayub A, et al. Evaluation of telephone and virtual visits for routine pediatric diabetes care during the COVID-19 pandemic. J Clin Transl Endocrinol 2020;22:100238.

18 Elbarbary NS, Dos Santos TJ, de Beaufort C, et al. COVID-19 outbreak and pediatric diabetes: perceptions of health care professionals worldwide. Pediatr Diabetes 2020;21:1083-92.

19 Sherifali D, Berard LD, Gucciardi E. Clinical Practice Guidelines for the Prevention and Management of Diabetes in Canada : Selfmanagement education and support. Canadian journal of diabetes 2018;2018:S36-41.

20 Dexcom clarity: Dexcom clarity diabetes management software 2016.

21 Urakami T. New insights into the pharmacological treatment of pediatric patients with type 2 diabetes. Clin Pediatr Endocrinol 2018;27:1-8.

22 Dougherty JP, Lipman TH, Hyams S, et al. Telemedicine for adolescents with type 1 diabetes. West $J$ Nurs Res 2014;36:1199-221.

23 McSwain SD, Bernard J, Burke BL, et al. American telemedicine association operating procedures for pediatric telehealth. Telemed $\mathrm{J}$ E Health 2017:23:699-706.

24 Lau N, Waldbaum S, Parigoris R, et al. eHealth and mHealth psychosocial interventions for youths with chronic illnesses: systematic review. JMIR Pediatr Parent 2020;3:e22329.

25 Cameron JD, Ramaprasad A, Syn T. An ontology of and roadmap for mHealth research. Int J Med Inform 2017;100:16-25.

26 Lewinski AA, Patel UD, Diamantidis CJ, et al. Addressing diabetes and poorly controlled hypertension: pragmatic $\mathrm{mHealth}$ selfmanagement intervention. J Med Internet Res 2019;21:e12541.

27 De Guzman KR, Snoswell CL, Taylor ML, et al. A systematic review of pediatric telediabetes service models. Diabetes Technol The 2020;22:623-38.

28 Berndt R-D, Takenga C, Preik P, et al. Impact of information technology on the therapy of type-1 diabetes: a case study of children and adolescents in Germany. J Pers Med 2014;4:200-17.

29 Moher D, Shamseer L, Clarke M, et al. Preferred reporting items for systematic review and meta-analysis protocols (PRISMA-P) 2015 statement. Syst Rev 2015;4:1.

30 Shamseer L, Moher D, Clarke M, et al. Preferred reporting items for systematic review and meta-analysis protocols (PRISMA-P) 2015: elaboration and explanation. BMJ 2015;349:97647.

31 Marshall WA, Tanner JM. Variations in pattern of pubertal changes in girls. Arch Dis Child 1969;44:291-303.

32 Marshall WA, Tanner JM. Variations in the pattern of pubertal changes in boys. Arch Dis Child 1970;45:13-23.

33 Granger D, Vandelanotte C, Duncan MJ, et al. Is preference for $\mathrm{mHealth}$ intervention delivery platform associated with delivery platform familiarity? BMC Public Health 2016;16:1-7.

34 Varni JW, Seid M, Kurtin PS. PedsQL 4.0: reliability and validity of the pediatric quality of life inventory version 4.0 generic core scales in healthy and patient populations. Med Care 2001;39:800-12.

35 Varni JW, Burwinkle TM, Jacobs JR, et al. The PedsQL in type 1 and type 2 diabetes: reliability and validity of the pediatric quality of life 
inventory generic core scales and type 1 diabetes module. Diabetes Care 2003;26:631-7.

36 Ingersoll GM, Marrero DG. A modified quality-of-life measure for youths: psychometric properties. Diabetes Educ 1991;17:114-8.

37 Dexcom. Dexcom G6 continuous glucose monitoring system 2018.

38 Massa GG, Gys I, Op 't Eyndt A, et al. Evaluation of the FreeStyle ${ }^{\circledR}$ Libre flash glucose monitoring system in children and adolescents with type 1 diabetes. Horm Res Paediatr 2018;89:189-99.

39 Imran SA, Rabasa-Lhoret R, Ross S. Targets for glycemic control. Can J Diabetes 2013;37:S31-4.

40 Bhatt M, Nahari A, Wang P-W, et al. The quality of clinical practice guidelines for management of pediatric type 2 diabetes mellitus: a systematic review using the agree II instrument. Syst Rev 2018;7:193.

41 Wolfsdorf Jl, Glaser N, Agus M, et al. ISPAD clinical practice consensus guidelines 2018: diabetic ketoacidosis and the hyperglycemic hyperosmolar state. Pediatr Diabetes 2018;19 Suppl 27:155-77.

42 Veritas Health Innovation. Covidence systematic review software. Australia: Veritas Health Innovation Melbourne, 2016.
43 Page MJ, McKenzie JE, Bossuyt PM, et al. The PRISMA 2020 statement: an updated guideline for reporting systematic reviews. BMJ 2021;372:n71.

44 Page MJ, Moher D, Bossuyt PM, et al. PRISMA 2020 explanation and elaboration: updated guidance and exemplars for reporting systematic reviews. BMJ 2021;372:n160.

45 Sterne JAC, Savović J, Page MJ, et al. Rob 2: a revised tool for assessing risk of bias in randomised trials. BMJ 2019;366:14898.

46 Sterne JA, Hernán MA, Reeves BC, et al. ROBINS-I: a tool for assessing risk of bias in non-randomised studies of interventions. BMJ 2016;355:i4919.

47 GRADE Working Group. Grading of recommendations assessment, development and evaluation, 2017. Available: gradeworkinggrouporg

48 Review Manager (RevMan) [Computer program]. Version 5.4, The Cochrane Collaboration 2020

49 Higgins JPT, Thomas J, Chandler J. Cochrane Handbook for systematic reviews of interventions version 6.1 (updated September 2020). Cochrane, 2020. Available: www.training.cochrane.org/ handbook

50 Williamson PR, Gamble C. Application and investigation of a bound for outcome reporting bias. Trials 2007;8:1-12. 